\title{
Estudio prospectivo del diámetro de la vía biliar principal antes y 12 años después de colecistectomía
}

\author{
Paula Csendes $\mathrm{G}^{1}$, Attila Csendes J ${ }^{2}$, Ana María Burgos L2 \\ Patricio Burdiles $\mathbf{P}^{2}$. \\ Bile duct diameter before and \\ 12 years after cholecystectomy
} common bile duct dilates after cholecystectomy. Aim: To measure common bile tract diameter before and 12 years after a cholecystectomy for cholelithiasis. Material and methods: Prospective study of 85 patients (aged 20 to 71 years, 68 fenmales) subjected to a cholecystectomy and followed for 12 years. All were asymptomatic and had abdominal ultrasound to measure common bile duct diameter, seven days before and 12 years after the surgical procedure. Results: Common bile duct diameter before and 12 years after surgery was $4.6 \pm 0.9$ and $5.0 \pm 1.8 \mathrm{~cm}$ respectively ( $\mathrm{p}=\mathrm{NS}$ ) among 69 patients aged less than 60 years. The figures for 16 patients aged more than 60 years were $5 \pm 0.8$ and $6.7 \pm 1.9(p<0.03)$. Conclusions: Among patients below 60 years of age there is no significant change in bile duct diameter 12 years after surgery. In subjects over 60 years of age there is a significant increase in this diameter (Rev Méd Chile 2007; 135: 735-42).

(Key words: Bile ducts, extrahepatic; Cholecystectomy; Ultrasonography)

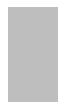

Recibido el 6 de abril, 2006. Aceptado el 10 de julio, 2006.

${ }^{1}$ Departamentos de Radiología y ${ }^{2}$ Cirugía, Hospital Clínico de la Universidad de Chile, Santiago, Chile.

$\mathrm{E}^{1}$ comportamiento del diámetro interno de la vía biliar principal (V.B.P.) (conductos hepáticos y colédoco) años después de colecistectomía, ha sido un motivo de controversia por mucho

Correspondencia a: Attila Csendes, MSCCh, FACS (Hon). Director, Departamento de Cinugía, Hospital Clínico Universidad de Chile. Santos Dumont 999. Santiago, Chile. Fono: 56-2-7774387. Fax: 56-2-7775043. E mail: acsendes@redclinicauchile.cl tiempo. Hay una opinión generalizada entre cirujanos y radiólogos, que la vía biliar principal se dilata después de una colecistectomía, debido a la pérdida de la función absorptiva de la vesícula biliar y al aumento de la presión intraluminal dentro del sistema ductal biliar. Esta dilatación fisiológica» fue enunciada por primera vez por Opie en $1887^{1}$. Posteriormente, esta hipótesis se confirmó por Judd y Mann², quienes encontraron en perros y gatos una dilatación uniforme de todos los conductos extrahepáticos después de 
colecistectomía. El mismo Judd comunicó, en 1923, a 24 pacientes con síndrome de postcolecistectomía»a quienes operó, encontrando dilatación de la V.B.P. sin evidencias de cálculos ${ }^{3}$. Hallazgos similares fueron publicados por Benson ${ }^{4}$. Desde entonces, diferentes autores han evaluado el diámetro de la V.B.P. antes y después de colecistectomía con diferentes métodos, reportando resultados diferentes.

El propósito del presente estudio prospectivo fue determinar el diámetro interno de la V.B.P. antes y 12 años posterior de haber realizado la colecistectomía por colecistolitiasis.

\section{MATERIAL Y MÉTODO}

Pacientes estudiados. Este estudio prospectivo comenzó en enero de 1987, cuando se diseñó un protocolo para análisis histológico, manométrico, bacteriológico y radiológico de la vía biliar en pacientes con colecistolitiasis o coledocolitiasis ${ }^{5-9}$. Como parte de este protocolo, se definió la medición del diámetro de la vía biliar principal antes y 12 años después de la cirugía, como asimismo la presencia de síntomas alejados de la colecistectomía ${ }^{10}$. Este protocolo se cerró en diciembre de 1991, cuando un total de 450 pacientes fueron evaluados en forma completa. De ellos, en 330 pacientes se había realizado colecistectomía sola y en 120 pacientes se había realizado, además, una exploración de V.B.P. De los 330 pacientes, se escogieron 1 de cada 4 pacientes al azar para el presente estudio, quienes fueron evaluados entre 1999 y 2004. Todos los pacientes habían sido sometidos a colecistectomía laparotómica y en ninguno hubo evidencia de cálculos de la V.B.P. tanto en la ecografía preoperatoria, durante la palpación manual intraoperatoria de la vía biliar y por colangiografía intraoperatoria en todos ${ }^{9}$. Por lo tanto, pacientes con cálculos de la V.B.P., historia de daño hepático previo o con pruebas hepáticas alteradas, ictericia, pancreatitis aguda o con dilatación de la V.B.P. mayor a $7 \mathrm{~mm}$ antes o durante la cirugía, se excluyeron del presente estudio.

Evaluación ultrasonográfica. Todos los pacientes incluidos en la presente investigación fueron evaluados por 2 radiólogos, 7 a 10 días antes de la colecistectomía, midiendo el diámetro interno de la V.B.P. en un equipo de tiempo real (Aloka Co, Japón). La evaluación alejada se realizó en todos los pacientes por un mismo radiólogo, con amplia experiencia en ultrasonografía de tiempo real (Aloka 1200, Co Japón), con un transductor de 3.5 MHZ. Después de un ayuno nocturno, los pacientes se evaluaron en inspiración profunda, ya sea en posición supina u oblicua lateral izquierda, con una aproximación subcostal derecha. La V.B.P. fue fácilmente identificada a nivel de la porta hepatis, donde la arteria hepática cursa perpendicular a ambas estructuras. La V.B.P. se midió en su porción media o suprapancreática, en un examen antero-posterior. Todas las mediciones se expresaron en $\mathrm{mm}$ y el error ultrasonográfico en la medición de la V.B.P. fue de $1 \pm \mathrm{mm}^{11-19}$.

Seguimiento. Todos los pacientes fueron interrogados y examinados personalmente por uno de los autores 12 años después de la cirugía. La ultrasonografía se realizó 3 a 5 días después de la entrevista.

Estadística. Todos los valores se expresaron en promedio \pm desviación estándar, señalando los valores mínimos y máximos en $\mathrm{mm}$. Para el análisis estadístico se emplearon el test de Chi cuadrado y el test de $\mathrm{T}$ de Student, tomando un $\mathrm{p}$ $<0,05$ como significativo.

\section{Resultados}

Los 85 pacientes incluidos en la presente investigación tenían, al momento de la colecistectomía,

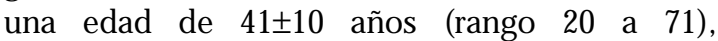
correspondiendo a 68 mujeres y 17 hombres. Hubo 69 pacientes menores de 60 años y 16 pacientes sobre 61 años. El promedio de seguimiento fue de 144 meses (rango 120 a 156). Todos estaban asintomáticos, ninguno había presentado dolor cólico o ictericia, la mayoría (96\%) había subido de peso y estaba ingiriendo dieta completa ${ }^{10}$. La Tabla 1 muestra el diámetro interno de la V.B.P. antes y 12 años después de la colecistectomía, de acuerdo a la edad en la operación original. Antes de la cirugía, no había diferencia significativa en el diámetro de la V.B.P. bajo 0 sobre 60 años $(p>0,1)$. En pacientes bajo 60 años, el diámetro de la V.B.P. aumentó en promedio $9 \%$ 


\section{Tabla 1. D iámetro interno de la vía biliar principal (en $\mathrm{mm}$ ) antes y 12 años después de colecistectomía de acuerdo a la edad en la operación original. $\mathbf{N}=85$}

\begin{tabular}{|lll|}
\hline & $\begin{array}{l}\leq 60 \text { años } \\
\mathrm{n}=69\end{array}$ & $\begin{array}{l}\geq 61 \text { años } \\
\mathrm{n}=16\end{array}$ \\
\hline $\begin{array}{l}\text { Preoperatorio } \\
\text { Promedio } \\
12 \text { años después colecistectomía } \\
\begin{array}{l}\text { Promedio } \\
\% \text { Aumento }\end{array}\end{array}$ & $5,62 \pm 0,89(\mathrm{~A})$ & $5,0 \pm 0,8(\mathrm{~B})$ \\
$\mathrm{p}$ AvB $=>0,1$ & $5,02 \pm 1,76(\mathrm{C})$ & $6,68 \pm 1,92(\mathrm{D})$ \\
CvD $=<0,001$ & 12 & 34 \\
AvC $=>0,09$ & & \\
BvD $=<0,003$ & & \\
\hline
\end{tabular}

a los 12 años de control, lo que no fue significativo $(p>0,09)$. Sin embargo, pacientes sobre 61 años mostraron un aumento significativo del diámetro de la V.B.P. en 34\%, comparado a las cifras preoperatorias $(p<0,003)$. Los valores promedios del diámetro de la V.B.P. después de la cirugía fueron significativamente mayores en pacientes sobre 61 años comparados al grupo de pacientes bajo 60 años $(\mathrm{p}<0,001)$.

La Tabla 2 muestra los cambios del diámetro interno de la V.B.P. doce años después de la colecistectomía. En pacientes bajo los 60 años, la mayoría (75\%) no mostró cambios en el diámetro de la V.B.P., mientras que $22 \%$ de los pacientes mostró un aumento entre 2 y $4 \mathrm{~mm}$. En pacientes sobre 61 años, sólo 2 pacientes (12\%) no mostraron cambios en el diámetro interno de la V.B.P. La mayoría $(62,5 \%)$ mostró un aumento del diámetro entre 2 y $6 \mathrm{~mm}$.

Tabla 2. Cambios en el diámetro de la vía biliar principal antes y 12 años después de colecistectomía $\mathbf{N}=85$

\begin{tabular}{|lccc|}
\hline & $\begin{array}{l}\leq 60 \text { años } \\
\mathrm{n}=69\end{array}$ & $\begin{array}{l}\geq 61 \text { años } \\
\mathrm{n}=16\end{array}$ & \\
\hline Sin cambio & $52(75,4 \%)$ & $4(25 \%)$ & \\
Aumento & $15(21,7 \%)$ & $10(62,5 \%)$ & \\
Disminución & $2(2,9 \%)$ & $2(12,5 \%)$ & \\
Aumento $\geq 3 \mathrm{~mm}$ & $8(11,6 \%)$ & $7(43,8 \%)$ & $\mathrm{p}<0,01$ \\
\hline
\end{tabular}

\section{DisCUSIÓN}

Los resultados del presente estudio muestran que en pacientes bajo 60 años al momento de la colecistectomía, no se aprecia un aumento significativo del diámetro interno de la V.B.P. 12 años después de la cirugía. Por el contrario, en pacientes sobre 61 años el aumento del diámetro interno de la V.B.P. fue significativo, probablemente debido en gran parte al efecto de la edad. El diámetro normal de la V.B.P. ha sido estudiado en extenso en varios estudios clásicos de autopsias ${ }^{20-23}$. En todos ellos, el diámetro interno muestra pocas variaciones a lo largo de la V.B.P. Este conducto biliar se divide en 2 porciones, que se relacionan mejor con la estructura, función e imagen radiológica $^{23}$, más que la clásica división anatómica del Testut.

a. Porción proximal que va desde la formación de la V.B.P. (hepático común) hasta 6 a $8 \mathrm{~cm}$ hacia distal hasta la porción retroduodenal, que tiene esencialmente un lumen uniforme, con un diámetro interno relativamente constante ${ }^{21-23}$ y b. Porción terminal corta, hasta la papila, con engrosamiento de la pared y lumen estrecho. Las mediciones reportadas en estas publicaciones se refieren al diámetro externo de la V.B.P., indicando un promedio de 7,4 $\mathrm{mm}$, valor enteramente similar a lo reportado por nosotros en mediciones intraoperatorias con un compás ${ }^{6}$ que demostró un diámetro interno de 7,4 $\mathrm{mm}$ en sujetos normales. La pared de la V.B.P. mide $1,1 \mathrm{~mm}$ en cada lado $^{23}$ lo que significa que el 
diámetro externo, corresponde, por lo tanto, a un valor promedio de $5 \mathrm{~mm}$. Este valor es muy cercano a lo determinado por diferentes mediciones ultrasonográficas $^{11-19}$ y al valor de nuestro grupo de pacientes con colecistolitiasis, quienes tuvieron un diámetro interno de 4,6 mm. El límite alto de lo normal para las mediciones ecográficas vańa entre 6 y $7 \mathrm{~mm}^{16-19}$. El efecto de la edad parece ser un factor importante en la determinación del diámetro de la V.B.P. ${ }^{14,15,24}$. Se ha postulado un aumento de 0,3 a $0,6 \mathrm{~mm}$ por década en sujetos sobre 50 años ${ }^{15,23,24}$. Estudios recientes ${ }^{17-19}$ han mostrado resultados similares. Perret et $\mathrm{al}^{17}$ realizaron un estudio en 1.018 pacientes entre 60 y 96 años. A los 60 años, el diámetro de la V.B.P. fue de 3,6 mm y a los 80 años de $4 \mathrm{~mm}(\mathrm{p}<0,009)$. Los valores máximos normales fluctuaron entre 6 y $7 \mathrm{~mm}$. Horrow et $\mathrm{al}^{18}$, en un análisis de 258 pacientes, demuestran una dilatación de la vía biliar de 0,1 $\mathrm{mm} / \mathrm{año}$, con un valor promedio de normales de $3,5 \pm 1,2 \mathrm{~mm}$. Bachar et $\mathrm{al}^{19}$, en un estudio prospectivo de 251 pacientes, demostraron que bajo los 5 años, el diámetro de la V.B.P. fue de $3,12 \mathrm{~mm}$ y sobre los 50 años de $4,19 \mathrm{~mm}$ ( $\mathrm{p}<0,01$ ), dilatándose esta vía biliar $0,04 \mathrm{~mm} /$ año. Por lo tanto, este aumento edad-dependiente no debe ser confundido con una dilatación fisiológica» de la V.B.P. después de colecistectomía, por lo que los valores deben expresarse por lo menos en 2 grupos, bajo y sobre los 60 años para expresar nuestros resultados.

La «dilatación fisiológica» de la V.B.P. es una opinión generalizada entre cirujanos y radiólogos, a menudo basada en pocas evidencias científicas ${ }^{1,23}$. Benson ${ }^{4}$ en un estudio de autopsia en 1940, concluyó que pacientes sasintomáticos» no presentaron dilatación de la V.B.P. después de colecistectomía, mientras que 14 pacientes con distress después de colecistectomía, tenían dilatación de la V.B.P. Obviamente, esto fue un estudio autópsico, no había mediciones preoperatorias y la definición de distress postcolecistectomía es difícil de entender. Posteriormente, Wise y cols ${ }^{25}$ realizaron colangiografías intravenosas en 142 pacientes sometidos a colecistectomía, todos «sintomáticos». Estos autores notaron una gran variación del diámetro de la V.B.P. de 3 a $30 \mathrm{~mm}$, teniendo la mayoría un diámetro cercano a $10 \mathrm{~mm}$.

Los estudios precisos partieron en 1957, cuando Qvist ${ }^{26}$ evaluó a pacientes con colangiografía intravenosa 1 año después de la cirugía, encontrando que en la mayoría, el diámetro de la V.B.P. no se modificaba, y sólo en pocos casos había una leve dilatación. En la Tabla 3 se resume los resultados de 19 publicaciones concernientes a mediciones del diámetro de la V.B.P. antes y después de colecistectomía ${ }^{26-43}$. La mitad de ellos son estudios prospectivos. Los métodos de determinación del diámetro de la V.B.P. han sido básicamente tres: colangiografía intravenosa en 9 reportes, colangiografía endoscópica retrograda en 1 y ultrasonografía en 10, incluyendo el presente estudio. Este hecho puede representar una importante fuente de error e inadecuada interpretación de los datos, debido a la irregular excreción y opacificación de la V.B.P. en el caso de la colangiografía intravenosa, a la mayor distensión de la V.B.P. en el caso de la colangiografía retrógrada y al hecho de sobservadordependiente» de los resultados con la ultrasonografía. Además, el sitio de medición del diámetro de la V.B.P. es importante y por lo tanto, las mediciones de diferentes estudios pueden haber sido en diferentes porciones de la V.B.P. Sin embargo, el consenso actual es considerar la ultrasonografía como el método de elección para determinar el diámetro de la V.B.P., debido a la facilidad para realizarla, el bajo costo, ausencia de complicaciones, no invasiva y posibilidad de repetir cuantas veces sea necesario.

Hammarstön, en una excelente revisión en $1997^{41}$, después de analizar 9 publicaciones, concluye que los resultados muestran en forma concluyente un aumento significativo (compensatorio y no relacionado con edad) del diámetro de la V.B.P. después de colecistectomía en la mayoría de los pacientes. Nuestros resultados no confirman esta hipótesis. El análisis de los datos publicados en los 19 trabajos, muestran que cuando se empleó colangiografía intravenosa, en 6 de 9 no hubo un aumento significativo del diámetro de la V.B.P. post colecistectomía ${ }^{26-29,31,32}$ y sólo en un reporte hubo un aumento significativo ${ }^{41}$. En 2 publicaciones no hubo análisis estadístico. El único trabajo que empleó una colangiografía retrógrada es difícil de analizar $^{38}$. Diez publicaciones, incluyendo la nuestra, han empleado la ultrasonografía. Cuatro de ellas informaron de un aumento significativo de la V.B.P. después de colecistectomía ${ }^{16,36,40,42}$ y cinco reportaron valores similares antes y después de colecistec- 


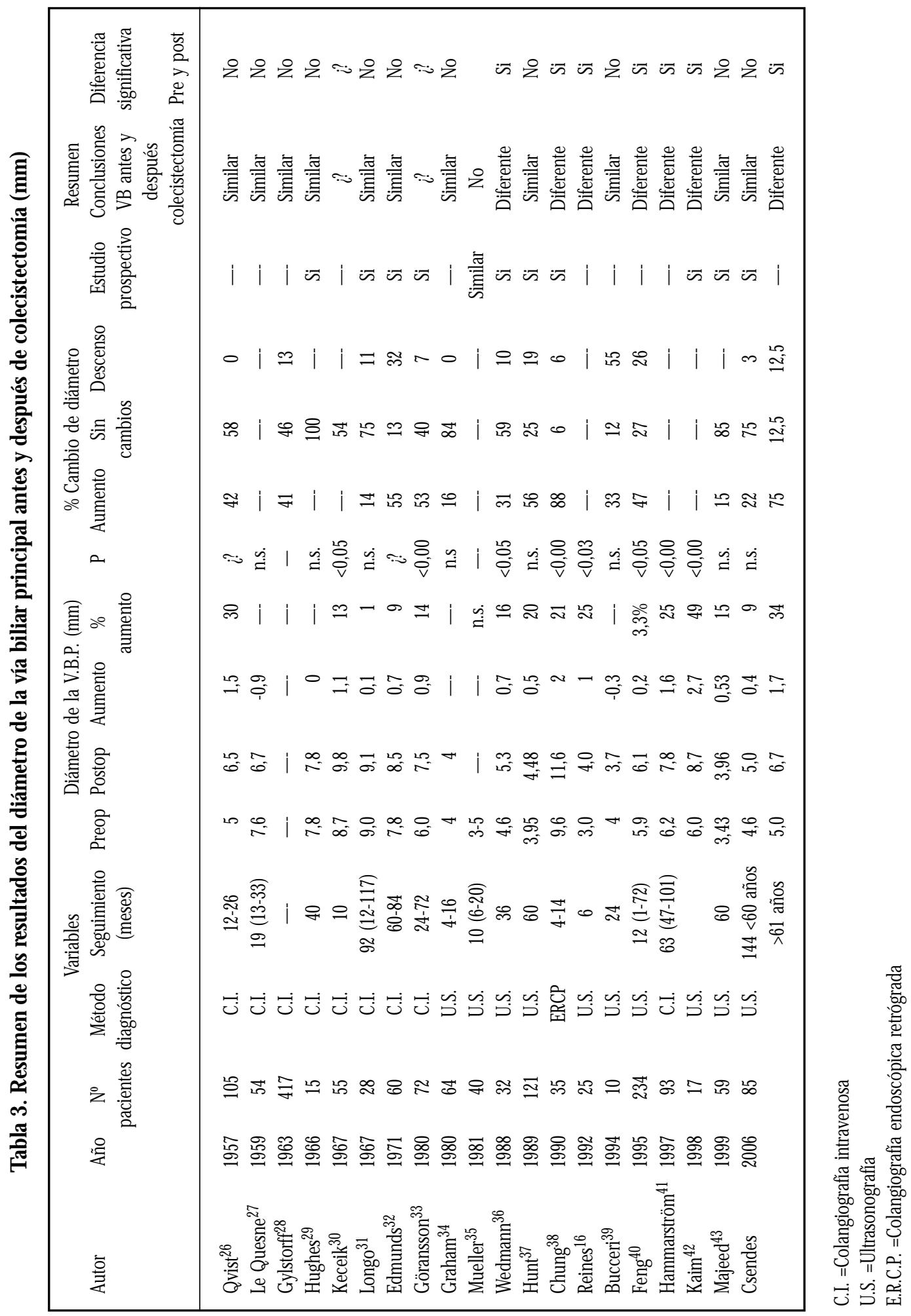


tomía $34,35,37,39,43$. Sin embargo, ninguno evaluó el efecto de la edad. Sabemos que lo más probable es que la colecistectomía se realiza usualmente en pacientes jóvenes, bajo los 60 años y por lo tanto pocos pacientes añosos se han evaluado. Por lo demás, en 14 (82\%) de 17 publicaciones, el seguimiento fue menor a 60 meses y sólo en $3(18 \%)$ este seguimiento fue mayor, hasta 90 meses. En 2 estudios no se reporta el seguimiento. Nuestro estudio es el que tiene el seguimiento más largo, de 144 meses. Por lo tanto, estos 2 hechos, edad joven y seguimiento corto, podrían explicar, en parte, la no dilatación de la V.B.P. post-colecistectomía. En el presente análisis, se quiso determinar la influencia de la edad sobre el diámetro de la V.B.P. En pacientes bajo 60 años no se observó una variación significativa, en cambio, sobre los 61 años, en la mayoría hubo un significativo aumento del diámetro de la V.B.P. Sin embargo, como las mediciones iniciales se realizaron hace más de una década y asumiendo un error de $1 \mathrm{~mm}$ en las mediciones, no podemos concluir enfáticamente que la dilatación de la V.B.P. sobre los 61 años se debe solamente a la cirugía y que no haya una influencia del envejecimiento. En todo caso, en nuestro estudio sólo se consideraron las variaciones de más de $2 \mathrm{~mm}$ en diámetro, mientras que una variación de $1 \mathrm{~mm}$ se consideró no variación. Se ha demostrado que con el aumento de la edad ${ }^{4}$, hay fragmentación de las pocas bandas de miocitos de musculatura lisa en la pared de la V.B.P., con aumento de tejido conectivo blando y disminución de las fibras elásticas, lo que explica en parte el aumento del diámetro interno de la V.B.P. con la edad. Todos estos pacientes estaban

\section{REFERENCIAS}

1. Oddi R. D'une disposition a sphincter speciale de l'ouverture du canal choledoque. Arch Ital Brol 1887; 8: 317-22.

2. Judd ES, MANN FC. The effect of remained of the gallbladder: an experimental study. Surg Gynec Obst 1917; 24: 437-42.

3. JudD ES. Condition of the common duct after cholecystectomy. JAMA 1923; 81: 704-9. asintomáticos, ya que las mediciones de la V.B.P. podían variar significativamente con la presencia de síntomas por coledocolitiasis residual o por disquinesia del esfínter de Oddi. El cuidadoso análisis de los 4 reportes que demuestran un aumento significativo del diámetro de la V.B.P. después de colecistectomía, muestran que este saumento» corresponde a $0,7 \mathrm{~mm}^{36}, 1,0 \mathrm{~mm}^{16}, 0,2 \mathrm{~mm}^{40}, 1,6 \mathrm{~mm}^{41}$, y 2,7 $\mathrm{mm}^{42}$. Asumiendo la influencia de la edad y del error de $1 \mathrm{~mm}$ en el observador, sólo 2 trabajos ${ }^{41,42}$ demostrańan un aumento significativo del diámetro interno de la V.B.P.

En resumen, la V.B.P. después de colecistectomía en pacientes bajo 60 años no muestra un cambio significativo del diámetro 12 años posterior a la cirugía, ya que el aumento de $0,4 \mathrm{~mm}$ en 12 años se puede explicar simplemente por el aumento normal que se aprecia de 0,4 a $0,6 \mathrm{~mm} /$ década. En pacientes sobre 61 años, hay un aumento significativo del diámetro intemo de la V.B.P. El aumento del diámetro de la V.B.P. de 30 más mm ocurrió sólo en $12 \%$ de los pacientes bajo 60 años y en $44 \%$ sobre los 61 años, lo que fue un valor estadísticamente significativo. El hecho que en casi la mitad de los pacientes sobre 60 años mostraron un aumento igual o mayor a $3 \mathrm{~mm}$, no se logra explicar solamente por efecto de la edad, ya que en 10 años este aumento no debiera ser mayor a 1 $\mathrm{mm}$. Por lo tanto, en este grupo de pacientes se aprecia también una dilatación fisiológica»y «compensatoria» de la vía biliar principal. No tenemos una clara explicación de por qué en $12 \%$ de los pacientes sobre 60 años la V.P.B. disminuye de diámetro 12 años después de colecistectomía.

4. Benson KW. Dilatation of the bile duct and its relation to distress after cholecystectomy. Am J Dig Dis 1940; 7: 1-3.

5. Maluenda $F$, Csendes A, Burdiles P, Díaz JC. Bacteriological studies of choledochal bile in patients with common bile duct stones with or without acute suppurative cholangitis. Hepatogastroenterology 1989; 36: 132-5.

6. Burdiles P, Csendes A, Díaz J, Smok G, Bastías J, Palominos G et al. Histological analysis of liver 
parenchymia and choledochal wall, and external diameter and intraluminal pressure of the common bile duct in controls and patients with common bile duct stones with or without acute suppurative cholangitis. Hepatogastroenterology 1989; 36: 143-6.

7. Csendes A, Burdiles P, Díaz J, Maluenda F, Ferrario M, Compan A. Bacteriological studies of liver parenchyma in controls and in patients with gallstones or common bile duct stones with or without acute cholangits. Hepatogastroenterology 1995; 42: 821-6.

8. Csendes A, Burdiles P, Maluenda F, Díaz J, Csendes P, Mitru N. Simultaneous bacteriologic assessment of bile from gallbladder and common bile duct in control subjects and patients with gallstones and common duct stones. Arch Surg 1996; 131: 389-94.

9. Csendes A, Burdiles P, Díaz JC, Maluenda F, Korn O, VALLejo E ET AL. Prevalence of common bile duct stones according to the increasing number of risk factors present. A prospective study employing routinely intraoperative cholangiography in 477 cases. Hepatogastroenterology 1998; 45: 1415-21.

10. Csendes A, Csendes P, Rojas J, Sánchez M. Análisis clínico de pacientes 10 años después de colecistectomía. Rev Méd Chile 2000; 128: 1309-12.

11. PARULEKAR SG. Ultrasound evaluation of common bile duct size. Radiology 1979; 133: 703-9.

12. Mueler PR, Ferucci JT, Simeone JF, VanSonnenberg E, HaLl DA, WitTernberg J. Observations on the distensibility of the common bile duct. Radiology 1982; 142: 467-72.

13. Niederau C, Mü山er J, Sonnenberg A. Extrahepatic bile ducts in healthy subjects, in patients with cholelithiasis and in postcholecytectomy patients. A prospective study. J Clin Ultrasound 1983; 11: 23-9.

14. Wu CC, Ho YH, Chen GY. Effect of aging on common bile duct diameter: a real time ultrasonographic study. J Clin Ultrasound 1984; 12: 473-8.

15. Chung-Chieng WU, Yan-Hui HO, Chang-Yi Chen. Effect of aging on common bile duct diameter: a real time ultrasonographic study. J Clin Ultrasound 1984; 12: 473-8.

16. Reines WR, Shady K, LND M, Scott R. Ultrasound evaluation of the common duct in symptomatic and asymptomatic patients. Am J Rad 1992; 87: 489-92.
17. Perret RS, Sloop GD, Borne JA. Common bile duct measurements in an elderly population. J Ultrasound Med 2000; 19: 727-30.

18. Horrow MM, Horrow JCH, Niakosari A, Kirby CL, ROSENBERG HK. Is age associated with size of adult extrahepatic bile duct: sonographic study. Radiology 2001; 221: 411-14.

19. Bachar GN, Cohen M, Belenky A, Atar E, Sideon $\mathrm{SH}$. Effect of aging on the adult extrahepatic bile duct. J Ultrasound Med 2003; 22: 879-82.

20. Dowdy GS, WaLdron GW, Brown WG. Surgical anatomy of the pancreato biliary ductal system. Arch Surg 1962; 84: 93-110.

21. HAND BH. Anatomical study of the choledochoduodenal area. BritJ Surg 1963; 50: 486-94.

22. Kune GA. Surgical anatomy of common bile duct. Arch Surg 1964; 89: 995-1004.

23. Mahour GH, WaKin KG, FerRIs DO. The common bile duct in man. Its diameter and circumference. Ann Surg 1967; 165: 415-19.

24. EDholm P, Jonsson G. Bile duct stones related to age and duct width. Acta Clin Scand 1962; 124: 75-9.

25. WISE RE, O'BRIEN RG. Interpretation of the intravenous cholangiogram. JAMA 1956; 160: 819-27.

26. QvisT CF. The influence of cholecystectomy on the common bile duct. Acta Chir Scand 1957; 113: 31-5.

27. Le Quesne LP, Whiteside CG, Hand BM. The common bile duct after cholecystectomy. Brit Med J 1959; I: 329-32.

28. GylstorfF J, FABer H. Choledochus changes after cholecystectomy (pre and postoperative examination in 400 patients). Acta Chir Scand 1963; 125: 499-501.

29. Hughes J, Lo Curcio SB, Edmunds R, FinBy N. The common duct after cholecystectomy. JAMA 1966; 197: 89-91.

30. Keclik M, Husralová H, Zeman J, Baudis J, Kavarova M. Gibt es eine Choledochuserweiterung nach der Cholezystektomie? Munich Med Wochensch 1967; 109: 418-23.

31. Longo ML, Hodgson JR, Ferris DO. Size of the common bile duct following cholecystectomy. Ann Surg 1967; 165: 250-3.

32. Edmunds R, Katz S, Garciano V, Finby N. The common duct after cholecystectomy. Arch Surg 1971; 103: 79-81.

33. GöRANSSON A. Cholegraphy: Its applicability and reliability in connection with gallstone operations. Acta Chir Scand 1980; supp 496: 1-95. 
34. Graham MF, Coopenberg PH, Cohen MM, Burhenne HJ. The size of the normal common hepatic duct following cholecystectomy: an ultrasonographic study. Radiology 1980; 135: 137-9.

35. Mueler PR, Ferrucci JT, Simeone JF, Wettenberg J, VanSonnenberg E, Polansky A et al. Postcholecystectomy bile duct dilatation: myth or reality? Am J Rad 1981; 136: 355-8.

36. Wedmann B, Börsch G, Coenen C, Paassen A. Effect of cholecystectomy on common bile duct diameters: a longitudinal prospective ultrasonographic study. J Clin Ultrasound 1988; 16: 619-24.

37. Hunt DR, Scott AJ. Changes in bile duct diameter after cholecystectomy: a 5 year prospective study. Gastroenterology 1989; 97: 1485-8.

38. Chung S, Leung J, Li A. Bile duct size after cholecystectomy: an endoscopic retrograde cholangiopancreatographic study. Brit J Surg 1990; 77: 534-5.
39. Bucceri AM, Brogma A, FerRar R. Common bile duct caliber following cholecystectomy: A two year sonographic survery. Abd Imag 1994; 19: 251-2.

40. FEng B, Song Q. Does the common bile duct dilate after cholecystectomy? Sonographic evaluation in 234 patients. Am J Rad 1995; 165: 859-61.

41. HAMMARSTRÖm LE. Is there a significant increase in bile duct width after cholecystectomy? Scand J Gastroent 1997; 32: 961-4.

42. Kaim A, Steinke K, Frank M, Enriquez R, Kirsch E, BOGARTZ G ET AL. Diameter of the common bile duct in the elderly patient: measurement by ultrasound. Eur Radiol 1998; 8: 1413-15.

43. MAJEED AW, Ross B, Johnson AG. The preoperative normal bile duct does not dilate after cholecystectomy: results of a five year study. Gut 1999; 45: 741-3.

44. Kiadian GP, AzNaurian AV. The age related characteristics of the muscular layer of the common bile duct in man. Morfología 1995; 108: 10-12. 